\title{
Evidence for the Benefits of Nonantipsychotic Pharmacological Augmentation in the Treatment of Depression
}

\author{
Chia-Ming Chang $\cdot$ Soichiro Sato $\cdot$ Changsu Han
}

Published online: 28 May 2013

(C) Springer International Publishing Switzerland 2013

\begin{abstract}
Failure to achieve an adequate response after initial antidepressant treatment in patients with depression is common and remains a clinical challenge. In recent years, some atypical antipsychotic agents have been approved by the US Food and Drug Administration for use in an augmentation strategy for major depressive disorder, and other agents are already in common use in clinical practice. We conducted a search of MEDLINE for relevant studies of augmentation strategies using randomized controlled trials and meta-analyses, and we summarize and discuss the various agents other than atypical antipsychotics. Lithium and thyroid hormone augmentation may improve the response of tricyclic antidepressants but not that of selective serotonin reuptake inhibitors. The efficacy of augmentation with modafinil, buspirone, methylphenidate, folic acid, pindolol and lamotrigine is limited or equivocal. Most of the studies have not focused on treatment-resistant depression (TRD). More trials are needed to
\end{abstract}

Previous Presentation: Part of the information presented in this article was presented at the round table discussion of psychiatrists from Asia and the USA, sponsored by Korea Otsuka International Asia and Arab.

C.-M. Chang ( $₫)$

Department of Psychiatry, Chang Gung Memorial Hospital at Linko and College of Medicine, Chang Gung University, Gweisan Township, 333 Taoyuan County,

Taiwan, Republic of China

e-mail: cmchang58@yahoo.com.tw

S. Sato

Zikei Hospital, Zikei Institute of Psychiatry, Okayama, Japan

C. Han

Department of Psychiatry College of Medicine,

Korea University, Seoul, Republic of Korea help develop evidence-based options for augmentation in TRD.

\section{Introduction}

Most patients with major depressive disorder (MDD) do not achieve an adequate response with first-line antidepressant monotherapy. A meta-analysis reviewed 182 randomized, double-blind, placebo controlled trials of antidepressants for adults with $\operatorname{MDD}(n=36,385)$, and the response rates for antidepressants and placebo were $53.8 \%$ and $37.3 \%$, respectively [1]. In the Sequenced Treatment Alternatives to Relieve Depression (STAR*D) study of patients with MDD, after 12 weeks of treatment with citalopram, only one-third of participants were in remission [2]. Patients with MDD without remission are disturbed by residual symptoms, cannot return to normal functioning, require increased utilization of medical services, and are vulnerable to relapse [3-5]. Treatment-resistant depression (TRD) is common and is a therapeutic challenge for clinicians and patients.

One of the major strategies in managing TRD is augmentation, which refers to the use of a psychotropic agent that is not indicated for depression to enhance the effect of an antidepressant. The advantage of augmentation is its potential for rapid improvement without the need for titration. However, augmentation also adds the complexity of polypharmacy to the treatment regimen, which can increase side effects and complicate adherence [6]. It is generally recommended if a partial response is achieved along with tolerability in a given treatment trial $[7,8]$.

In the past decade, some atypical antipsychotics such as an olanzapine-fluoxetine combination, aripiprazole and quetiapine have been approved by the US Food and Drug 
Administration for the adjunctive therapy of MDD [9]. However, the potential side effects, including sedation, akathisia, weight gain, Q-T prolongation and metabolic syndrome should be noted [10]. For over 30 years, despite a dearth of evidence and off-label indications, some other agents have been used in clinical practice for augmentation in the treatment of MDD [11, 12], and these are still listed in several clinical guidelines [13-16].

The Canadian Network for Mood and Anxiety Treatment (CANMAT) clinical guidelines for the management of depressive disorder in adults [14] recommend different strategies (switch or add-on) and different agents (e.g. atypical antipsychotics and lithium) at different stages (first, second, or third-line treatment) based on the level of available evidence, as follows: Level 1: at least two randomized controlled trials (RCTs) with adequate sample sizes, preferably placebo controlled, and/or meta-analysis with narrow confidence intervals; Level 2: at least one RCT with adequate sample size and/or meta-analysis with wide confidence intervals; Level 3: nonrandomized, controlled prospective studies, case series, or high-quality retrospective studies; and Level 4: expert opinion/consensus (Table 1). It should be noted that the level of evidence merely represents the quality and nature of the studies that have been conducted. The purpose of this paper is to review and discuss the various agents other than atypical antipsychotics in the augmentation of antidepressants for MDD by their levels of evidence.

\section{Methods of Literature Review}

We searched MEDLINE (1975-2011) to identify RCTs and meta-analyses to evaluate various agents other than atypical antipsychotics in augmentation for MDD. We used 'augmentation', 'adjunctive', 'depression', 'antidepressant' and 'treatment refractory depression' as the keywords. No language constraints were applied. We reviewed the relevant literature by level of evidence.

\section{Specific Agent for Augmentation Therapy for Major Deprissive Disorder by Level of Evidence}

\subsection{Level 1: Lithium}

Since 1981, lithium augmentation has been used to enhance the effects of antidepressants [17].

Lithium augmentation of the tricyclic antidepressants (TCAs) has been studied extensively. A formal metaanalysis of 10 placebo controlled, double-blind studies ( $n=269$ ) found that lithium was significantly superior to placebo (mean response rate $41 \%$ versus $14 \%, p<0.001$ ) in the augmentation of antidepressants, including TCAs and selective serotonin reuptake inhibitors (SSRIs) [18].

Crossley and Bauer (2007) [19] have undertaken two meta-analyses to review five acceleration studies (231 participants) adding lithium to TCA and 10 augmentation studies (269 participants) adding lithium to various antidepressants including SSRIs. In the acceleration metaanalysis, a statistical trend in favour of lithium was found [standardized mean difference of $-0.43,95 \%$ confidence interval (CI) -0.93 to 0.07$]$. In the augmentation metaanalysis, lithium was significantly more effective than placebo [odds ratio $(\mathrm{OR})=3.11,95 \%$ CI 1.80 to 5.37]. Forty per cent of study participants on lithium responded against $17 \%$ on placebo. However, many of the studies included in this meta-analysis were small. One study had 61 patients and another had 35 or fewer patients. Usual doses of lithium during augmentation were 600-900 mg/ day, which translates into a lithium level of $0.4 \mathrm{mEq} / \mathrm{L}$ or greater, which is lower than the therapeutic level.

Bauer and colleagues [20] completed a recent metaanalysis in 2010. However, few studies were conducted on SSRI nonresponders. Most of those studies were open label, and the results confirmed that augmentation with lithium was effective in TRD (Table 2) [20-30].

Lithium remains a first-line augmentation strategy best supported by double-blind, placebo controlled RCTs. It is effective as both a response modulator and accelerant, often effecting a treatment response within 2 weeks of beginning treatment at doses between 600 and $900 \mathrm{mg} /$ day, corresponding to serum levels greater than $0.5 \mathrm{mEq} / \mathrm{L}$. The disadvantages of lithium use include an uptitration period, with up to 6 weeks needed for response, and the need for blood drug-level monitoring.

Lithium is one of the oldest and most studied agents used to improve outcomes in patients who experience inadequate results from an antidepressant trial. The evidence is strongest for patients whose depression is inadequately treated by an initial trial of a TCA. However, the data for augmentation of newer antidepressants are weak, and significant benefit has not been observed in the two most recent trials of this strategy [29, 30]. The low doses of lithium used (the mean plasma concentration was only $0.25 \mathrm{mEq} / \mathrm{L}$ ) [29] may explain why there was no significant difference between lithium and placebo augmentation to the TCA. Another trial studied depressed patients who had already received one or more adequate trials of therapy with SSRIs before the sequenced combination of nortriptyline. After 6 weeks of double-blind augmentation, no significant difference was seen between lithium and placebo augmentation ( $12.5 \%$ of subjects responded to lithium and $20.0 \%$ to placebo) [30].

TCAs are now typically reserved for patients with more advanced levels of treatment resistance; thus the relevance 
Table 1 Recommendations for nonresponse and incomplete response to an initial antidepressant by Canadian Mood and Anxiety Treatment guidelines

\begin{tabular}{|c|c|c|}
\hline \multirow[t]{10}{*}{ First-line } & $\begin{array}{l}\text { Switch to an agent with } \\
\text { evidence of superiority }\end{array}$ & Milnacipran (level 2) \\
\hline & & Duloxetine (level 2) \\
\hline & & Escitalopram (level 1) \\
\hline & & Mirtazapine (level 2) \\
\hline & & Sertraline (level 1) \\
\hline & & Venlafaxine (level 1) \\
\hline & Add-on another agent & Aripiprazole (level 1) \\
\hline & & Lithium (level 1) \\
\hline & & Olanzapine (level 1) \\
\hline & & Risperidone (level 2) \\
\hline \multirow[t]{8}{*}{ Second-line } & Add-on another agent & Bupropion (level 2) \\
\hline & & $\begin{array}{l}\text { Mirtazapine/mianserin } \\
\quad(\text { level 2) }\end{array}$ \\
\hline & & Quetiapine (level 2) \\
\hline & & Triiodothyronine (level 2) \\
\hline & & $\begin{array}{l}\text { Other antidepressant } \\
\text { (level 3) }\end{array}$ \\
\hline & $\begin{array}{l}\text { Switch to an agent with } \\
\text { evidence of superiority }\end{array}$ & Amitriptyline (level 2) \\
\hline & & Clomipramine (level 2) \\
\hline & & MAOI (level 2) \\
\hline \multirow[t]{4}{*}{ Third-line } & Add-on another agent & Buspirone (level 2) \\
\hline & & Modafinil (level 2) \\
\hline & & Stimulants (level 3) \\
\hline & & Ziprasidone (level 3) \\
\hline
\end{tabular}

Adapted from Lam et al. [14]

MAOI monoamine oxidase inhibitor

of lithium augmentation in contemporary practice is less clear than might be assumed from the strong recommendations found in current guidelines. Given the side effects, relatively low therapeutic index, and longer-term risks of thyroid and renal compromise associated with this agent, it should be used to augment SSRIs with caution.

\subsection{Level 2: Triiodothyronine}

Thyroid hormone augmentation is another common option. It has been shown that L-triiodothyronine (T3) is superior in efficacy to thyroxine (T4) in TRD [31].

A meta-analysis was performed by Aronson et al. (1996) [32] to assess the efficacy of augmentation with $\mathrm{T} 3$ in patients with MDD refractory to TCAs. They found that pooled effects across the four randomized double-blind studies were not significant (relative response 1.53; $95 \%$ CI 0.70 to $3.35 ; p=0.29$ ), but one study with negative results accounted for most of this result.
Table 2 Results of randomized, placebo-controlled lithium augmentation studies in depression

\begin{tabular}{lrll}
\hline Authors (year) & N & Study length & Efficacy \\
\hline Initial with TCA & & & \\
Heninger et al. (1983) [20] & 21 & 2 weeks & Lithium $>$ placebo \\
Kantor et al. (1986) [21] & 7 & 2 days & Lithium=placebo \\
Zusky et al. (1988) [22] & 16 & 3 weeks & Lithium=placebo \\
Schopf et al. (1989) [23] & 27 & 1 week & Lithium $>$ placebo \\
Browne et al. (1990) [24] & 17 & 2 days & Lithium=placebo \\
Stein and Bernadt (1993) & 34 & 6 weeks & Lithium $>$ placebo \\
[29] & & & \\
Joffe et al. (1993) [25] & 50 & 2 weeks & Lithium $>$ placebo \\
Nierenberg et al. (2003) & 35 & 6 weeks & Lithium=placebo \\
[30] & & & \\
Initial with SSRI & 61 & 6 weeks & Lithium $>$ placebo \\
Katona (1995) [26] & 24 & 1 week & Lithium $>$ placebo \\
Baumann (1993) [27] & & \\
\hline $\begin{array}{l}\text { Adapted from Bauer et al. [28] } \\
\text { SSRI selective serotonin } \\
\text { antidepressant reuptake inhibitor, }\end{array}$ & TCA tricyclic
\end{tabular}

Another meta-analysis by Altshuler et al. [33] found that in five of six studies, T3 was significantly more effective than placebo in accelerating clinical response to TCAs in nonrefractory depression (weighted effect size index $=$ 0.58). They also found that women were more likely than men to benefit from this strategy.

Previous studies have focused on the augmentation of T3 in TCA-refractory depression; therefore, Cooper-Kazaz and colleagues [34] conducted a meta-analysis on five RCTs; three were enhancement studies in which T3 was administered concurrently with the SSRIs from the start of treatment, and two were augmentation studies in which T3 was added for patients who had not responded to SSRI treatment. They found that the two open augmentation studies were too disparate in methodology to allow metaanalysis. One large RCT showed some support for an effect of T3 in SSRI nonresponsive patients; a smaller, underpowered RCT showed no benefit. The three enhancement studies were inconclusive in that one showed strongly positive effects of $\mathrm{T} 3$, one showed no effect, and one showed a trend.

The STAR*D trial compared remission rates for patients with TRD who were randomly assigned to lithium or T3 augmentation and found a trend in favour of T3 $(25 \%)$ over lithium (16\%), although the difference was not significant [35].

The dose of T3 for augmentation was typically $25-50 \mu \mathrm{g} /$ day, which was similar to the STAR*D study. To date, T3 augmentation has established efficacy for TCA 
nonresponders, but whether T3 augmentation is effective in SSRI-resistant patients is still unknown.

T3 augmentation is well tolerated and common side effects include palpitations, sweating, nervousness and tremor.

\subsection{Level 2: Modafinil}

Modafinil is a novel stimulant with pharmacological actions distinct from those of conventional amphetamines. DeBattista et al. [36] conducted a 6-week, double-blind, placebo controlled study in 136 MDD patients who had a partial response to antidepressant treatment. Patients received once-daily doses (100-400 mg) of modafinil or matched placebo as adjunct treatment with ongoing antidepressant therapy. Compared with placebo, modafinil resulted in a significantly greater mean improvement from baseline in fatigue (Fatigue Severity Index) at week 2 $(p<0.005)$ and sleepiness at week $1(p<0.01)$, but no effect on either parameter at the study endpoint.

Fava et al. [37] conducted an 8-week trial in 311 SSRI partial responders with excessive fatigue and sleepiness who were randomly assigned to either $200 \mathrm{mg} /$ day modafinil or placebo. Although there were no significant differences between the placebo and modafinil groups on two fatigue scales at the final visit, global functioning was improved in the modafinil trial group.

Those studies support the idea that modafinil at $100-400 \mathrm{mg} /$ day could act as an accelerant or adjunct to antidepressant treatment in relation to some depression symptoms, such as fatigue and sleepiness, but not to core symptoms such as mood and anhedonia. They also suggest that modafinil might be more appropriate as an augmentation agent in the treatment of antidepressant partial responders with excessive fatigue.

\subsection{Level 2: Buspirone}

Buspirone is an anxiolytic medication that acts as a partial serotonin 1A receptor agonist. Buspirone was first proposed for use in augmentation with SSRIs in 1991 [38]. The addition of buspirone to an SSRI or TCA may enhance the serotinergic effect and improve depressive symptoms.

The STAR*D trial demonstrated that augmentation with buspirone did not differ significantly from augmentation with bupropion following the failure of monotherapy with SSRI (remission rate 29.1 \%: 30.1\%) [39]. Two controlled studies [40, 41] and one open study [42] found no effect of buspirone as augmentation therapy in TRD.

\subsection{Level 3: Pindolol}

Low-dose pindolol is a relatively potent serotonin $1 \mathrm{~A}$ receptor antagonist. A meta-analysis conducted by
Ballesteros and Callado [43] reviewed 15 clinical trials of pindolol added to antidepressants (SSRI, TCA, or trazodone). They found that adjunctive therapy with pindolol could significantly accelerate the response to the antidepressants, but the ultimate effect on treatment outcome was no greater than that of an antidepressant plus placebo.

\subsection{Level 3: Methylphenidate}

Methylphenidate is typically used in the treatment of patients with attention deficit hyperactivity disorder. Two RCTs of augmentation with methylphenidate failed to detect differences in outcomes compared with placebo [44, 45].

A 4-week, double-blind, placebo controlled trial was conducted by Pae and colleagues [46], but they failed to detect a significant benefit of applying methylphenidate extended-release (OROS MPH) to improve antidepressantrelated sexual dysfunction in patients with TRD.

\subsection{Level 3: Omega-3 Fatty Acid}

A meta-analysis [47] was conducted on 13 placebo controlled RCTs of omega-3 fatty acid treatment of MDD. Only six of them were augmentation therapy, and the other seven studies were monotherapy. There was no significant benefit of omega-3 fatty acid treatment compared with placebo.

Lin and $\mathrm{Su}$ [48] conducted a meta-analysis of seven placebo controlled trials of the antidepressant efficacy of omega-3 fatty acids. They found a significant antidepressant effect of omega-3 polyunsaturated fatty acids, but publication bias and heterogeneity may have accounted for this result. One trial was monotherapy, two trials studied bipolar depressed patients and none of them studied TRD patients.

The advantages of omega- 3 fatty acids are their tolerability and acceptability, and they may possess other healthpromoting benefits. The disadvantages are the unknown optimal dose [1 g/day eicosapentaenoic acid (EPA) plus docosahexaenoic acid] and cost [49]. A meta-analysis was conducted by Sublette et al. [50], who found that if omega3 was greater than $60 \%$ EPA trials were significantly effective but if EPA was less than $60 \%$ it was not effective in the treatment of depression. Given that the diet in Asia may be rich in omega-3, whether omega-3 is less useful in these areas is still unknown.

\subsection{Level 3: Folic Acid}

Folic acid has been primarily assessed as a predictor of antidepressant response and as an adjunctive treatment. Low folic acid blood levels have been associated with lack 
of response and slower response to fluoxetine for MDD [51], and higher folic acid levels at treatment baseline are associated with a better response to antidepressants [52].

A review [53] found that folic acid had the potential to enhance the response of patients with depression. Folic acid has been compared with placebo as an adjunctive treatment combined with fluoxetine, with a significantly greater improvement in those receiving folic acid, especially among female patients [54]. It has also been noted to have advantages in terms of a decreased incidence of birth defects [55].

\subsection{Level 3: Anticonvulsants}

Although their use in this context has not been extensively evaluated, anticonvulsants such as carbamazepine, valproic acid and lamotrigine may offer some benefit in the treatment of TRD [39, 56].

Thomas and colleagues [57] have reviewed studies of augmentation with lamotrigine for the treatment of TRD patients who have failed treatment with at least one antidepressant. They found that lamotrigine was significantly superior to placebo on the Clinical Global Impression Scale for severity and improvement; however, this was not the case on the Hamilton Rating Scale for Depression (primary endpoint) and the Montgomery-Åsberg Depression Rating Scale.

\section{Conclusions and Future Directions}

This paper reviews the relevant literature about augmentation therapy with psychotropic agents other than atypical antipsychotics in major depression. Lithium and T3 are preferred agents to augment TCA, but not SSRI, nonresponse. The efficacy of augmentation with modafinil, buspirone, methylphenidate, folic acid, pindolol and lamotrigine is limited or equivocal. Most studies were uncontrolled and open-labelled, with small sample sizes and evaluated augmentation with TCAs, which have now been replaced by SSRIs as first-line therapy for MDD. Some studies were not designed for TRD patients. Therefore, it is not known whether they can be generalized for the treatment of MDD with initial SSRI failure. To investigate their efficacy and safety in the augmentation of SSRI, additional well-designed, randomized, placebo controlled trials should be conducted.

Acknowledgments Although Korea Otsuka International Asia and Arab (KOIAA) was involved in supporting the production of the supplement, the content of the manuscript, its review and revision, and the decision to submit to CNS Drugs were made solely by the authors and the supplement guest editor.

Sources of Financial Support The authors did not receive honorarium for writing this manuscript.
Conflict of interest Drs Chang and Sato have received honoraria from Korea Otsuka International Asia and Arab. Dr Han has received research grants from GlaxoSmithKline Korea, Eisai Korea, Korea Otsuka International Asia and Arab, Hanlim Pharmaceutical, Janssen Pharmaceuticals Korea, Eli Lilly and Co. Korea, Korean Health Technology R\&D Project, Ministry of Health and Welfare, Republic of Korea, Korean Research Foundation, and Otsuka Korea. Dr Han has also received honoraria from GlaxoSmithKline Korea, Lundbeck Korea, AstraZeneca Korea, Janssen Pharmaceuticals Korea, Eisai Korea, Abott Korea, Eli Lilly and Company Korea, Norvatis Korea and Otsuka Korea.

Disclosure This manuscript has been published in a journal supplement that was created with an unrestricted educational grant from Korea Otsuka International Asia and Arab (KOIAA).

\section{References}

1. Papakostas GI, Fava M. Does the probability of receiving placebo influence clinical trial outcome? A meta-regression of doubleblind, randomized clinical trials in MDD. Eur Neuropsychopharmacol. 2009;19(1):34-40.

2. Rush AJ, et al. STAR*D: revising conventional wisdom. CNS Drugs. 2009;23(8):627-47.

3. Nierenberg AA, et al. Residual symptoms after remission of major depressive disorder with citalopram and risk of relapse: a STAR*D report. Psychol Med. 2010;40(1):41-50.

4. Paykel ES, et al. Residual symptoms after partial remission: an important outcome in depression. Psychol Med. 1995;25(6): $1171-80$

5. Thase ME. Evaluating antidepressant therapies: remission as the optimal outcome. J Clin Psychiatry. 2003;64(Suppl. 13): $18-25$.

6. Philip NS, et al. Pharmacologic approaches to treatment resistant depression: a re-examination for the modern era. Expert Opin Pharmacother. 2010;11(5):709-22.

7. Shelton RC, et al. Therapeutic options for treatment-resistant depression. CNS Drugs. 2010;24(2):131-61.

8. Zaratiegui R. STAR*D study: searching strategies regarding lack of response to an SSRI. Vertex. 2009;20(84):119-28.

9. Connolly KR, Thase ME. If at first you don't succeed: a review of the evidence for antidepressant augmentation, combination and switching strategies. Drugs. 2011;71(1):43-64.

10. Chen J, Gao K, Kemp DE. Second-generation antipsychotics in major depressive disorder: update and clinical perspective. Curr Opin Psychiatry. 2011;24(1):10-7.

11. Dording CM, et al. The pharmacologic management of SSRIinduced side effects: a survey of psychiatrists. Ann Clin Psychiatry. 2002;14(3):143-7.

12. Mischoulon D, et al. Strategies for managing depression refractory to selective serotonin reuptake inhibitor treatment: a survey of clinicians. Can J Psychiatry. 2000;45(5):476-81.

13. American Psychiatric Association. Practice guideline for the treatment of patients with major depressive disorder (revision). Am J Psychiatry. 2000;157(4 Suppl.):1-45.

14. Lam RW, et al. Canadian Network for Mood and Anxiety Treatments (CANMAT) clinical guidelines for the management of major depressive disorder in adults. III. Pharmacotherapy. J Affect Disord. 2009;117(Suppl. 1):S26-43.

15. Bauer $M$, et al. World Federation of Societies of Biological Psychiatry (WFSBP) guidelines for biological treatment of unipolar depressive disorders in primary care. World J Biol Psychiatry. 2007;8(2):67-104. 
16. Spijker J, Nolen WA. An algorithm for the pharmacological treatment of depression. Acta Psychiatr Scand. 2010;121(3): $180-9$.

17. De Montigny C, et al. Lithium induces rapid relief of depression in tricyclic antidepressant drug non-responders. Br J Psychiatry. 1981;138:252-6.

18. Bauer M, Dopfmer S. Lithium augmentation in treatment-resistant depression: meta-analysis of placebo-controlled studies. J Clin Psychopharmacol. 1999;19(5):427-34.

19. Crossley NA, Bauer M. Acceleration and augmentation of antidepressants with lithium for depressive disorders: two metaanalyses of randomized, placebo-controlled trials. J Clin Psychiatry. 2007;68(6):935-40.

20. Heninger GR, Charney DS, Sternberg DE. Lithium carbonate augmentation of antidepressant treatment. An effective prescription for treatment-refractory depression. Arch Gen Psychiatry. 1983;40(12):1335-42.

21. Kantor D, et al. The benefit of lithium carbonate adjunct in refractory depression — fact or fiction? Can J Psychiatry. 1986;31(5): 416-8.

22. Zusky PM, et al. Adjunct low dose lithium carbonate in treatment-resistant depression: a placebo-controlled study. J Clin Psychopharmacol. 1988;8(2):120-4.

23. Schopf J, et al. Treatment of endogenous depressions resistant to tricyclic antidepressants or related drugs by lithium addition. Results of a placebo-controlled double-blind study. Pharmacopsychiatry. 1989;22(5):183-7.

24. Browne M, et al. Lithium as an adjunct in the treatment of major depression. Int Clin Psychopharmacol. 1990;5(2):103-10.

25. Joffe RT, et al. A placebo-controlled comparison of lithium and triiodothyronine augmentation of tricyclic antidepressants in unipolar refractory depression. Arch Gen Psychiatry. 1993;50(5): 387-93.

26. Katona CL, et al. Placebo-controlled trial of lithium augmentation of fluoxetine and lofepramine. Br J Psychiatry. 1995;166(1): 80-6.

27. Baumann P, et al. A double-blind, placebo-controlled study of citalopram with and without lithium in the treatment of therapyresistant depressive patients: a clinical, pharmacokinetic, and pharmacogenetic investigation. J Clin Psychopharmacol. 1996;16(4): 307-14.

28. Bauer M, et al. Lithium's emerging role in the treatment of refractory major depressive episodes: augmentation of antidepressants. Neuropsychobiology. 2010;62(1):36-42.

29. Stein G, Bernadt M. Lithium augmentation therapy in tricyclicresistant depression. A controlled trial using lithium in low and normal doses. Br J Psychiatry. 1993;162:634-40.

30. Nierenberg AA, et al. Lithium augmentation of nortriptyline for subjects resistant to multiple antidepressants. J Clin Psychopharmacol. 2003;23(1):92-5.

31. Joffe RT, Singer W. A comparison of triiodothyronine and thyroxine in the potentiation of tricyclic antidepressants. Psychiatry Res. 1990;32(3):241-51.

32. Aronson R, et al. Triiodothyronine augmentation in the treatment of refractory depression. A meta-analysis. Arch Gen Psychiatry. 1996;53(9):842-8.

33. Altshuler LL, et al. Does thyroid supplementation accelerate tricyclic antidepressant response? A review and meta-analysis of the literature. Am J Psychiatry. 2001;158(10):1617-22.

34. Cooper-Kazaz R, Lerer B. Efficacy and safety of triiodothyronine supplementation in patients with major depressive disorder treated with specific serotonin reuptake inhibitors. Int J Neuropsychopharmacol. 2008;11(5):685-99.

35. Nierenberg AA, et al. A comparison of lithium and T(3) augmentation following two failed medication treatments for depression: a STAR*D report. Am J Psychiatry. 2006;163(9): 1519-30; quiz 1665 .

36. DeBattista C, et al. Adjunct modafinil for the short-term treatment of fatigue and sleepiness in patients with major depressive disorder: a preliminary double-blind, placebo-controlled study. J Clin Psychiatry. 2003;64(9):1057-64.

37. Fava M, Thase ME, DeBattista C. A multicenter, placebo-controlled study of modafinil augmentation in partial responders to selective serotonin reuptake inhibitors with persistent fatigue and sleepiness. J Clin Psychiatry. 2005;66(1):85-93.

38. Jacobsen FM. Possible augmentation of antidepressant response by buspirone. J Clin Psychiatry. 1991;52(5):217-20.

39. Trivedi $\mathrm{MH}$, et al. Medication augmentation after the failure of SSRIs for depression. N Engl J Med. 2006;354(12):1243-52.

40. Landen M, et al. A randomized, double-blind, placebo-controlled trial of buspirone in combination with an SSRI in patients with treatment-refractory depression. J Clin Psychiatry. 1998;59(12): 664-8.

41. Appelberg BG, et al. Patients with severe depression may benefit from buspirone augmentation of selective serotonin reuptake inhibitors: results from a placebo-controlled, randomized, doubleblind, placebo wash-in study. J Clin Psychiatry. 2001;62(6): 448-52.

42. Onder E, Tural U. Faster response in depressive patients treated with fluoxetine alone than in combination with buspirone. J Affect Disord. 2003;76(1-3):223-7.

43. Ballesteros J, Callado LF. Effectiveness of pindolol plus serotonin uptake inhibitors in depression: a meta-analysis of early and late outcomes from randomised controlled trials. J Affect Disord. 2004;79(1-3):137-47.

44. Patkar AA, et al. A randomized, double-blind, placebo-controlled trial of augmentation with an extended release formulation of methylphenidate in outpatients with treatment-resistant depression. J Clin Psychopharmacol. 2006;26(6):653-6.

45. Ravindran AV, et al. Osmotic-release oral system methylphenidate augmentation of antidepressant monotherapy in major depressive disorder: results of a double-blind, randomized, placebo-controlled trial. J Clin Psychiatry. 2008;69(1):87-94.

46. Pae CU, et al. Methylphenidate extended release (OROS MPH) for the treatment of antidepressant-related sexual dysfunction in patients with treatment-resistant depression: results from a 4-week, double-blind, placebo-controlled trial. Clin Neuropharmacol. 2009;32(2):85-8.

47. Bloch MH, Hannestad J. Omega-3 fatty acids for the treatment of depression: systematic review and meta-analysis. Mol Psychiatry. 2011.

48. Lin PY, Su KP. A meta-analytic review of double-blind, placebocontrolled trials of antidepressant efficacy of omega- 3 fatty acids. J Clin Psychiatry. 2007;68(7):1056-61.

49. Papakostas GI. Managing partial response or nonresponse: switching, augmentation, and combination strategies for major depressive disorder. J Clin Psychiatry. 2009;70(Suppl. 6):16-25.

50. Sublette ME, et al. Meta-analysis of the effects of eicosapentaenoic acid (EPA) in clinical trials in depression. J Clin Psychiatry. 2011;72(12):1577-84.

51. Papakostas GI, et al. Serum folate, vitamin B12, and homocysteine in major depressive disorder. Part 1: predictors of clinical response in fluoxetine-resistant depression. J Clin Psychiatry. 2004;65(8):1090-5.

52. Alpert M, Silva RR, Pouget ER. Prediction of treatment response in geriatric depression from baseline folate level: interaction with an SSRI or a tricyclic antidepressant. J Clin Psychopharmacol. 2003;23(3):309-13.

53. Lazarou C, Kapsou M. The role of folic acid in prevention and treatment of depression: an overview of existing evidence and 
implications for practice. Complement Ther Clin Pract. 2010;16(3):161-6.

54. Coppen A, Bailey J. Enhancement of the antidepressant action of fluoxetine by folic acid: a randomised, placebo controlled trial. J Affect Disord. 2000;60(2):121-30.

55. Green NS. Folic acid supplementation and prevention of birth defects. J Nutr. 2002;132(8 Suppl.):2356S-60S.
56. Fava M, Rush AJ. Current status of augmentation and combination treatments for major depressive disorder: a literature review and a proposal for a novel approach to improve practice. Psychother Psychosom. 2006;75(3):139-53.

57. Thomas SP, Nandhra HS, Jayaraman A. Systematic review of lamotrigine augmentation of treatment resistant unipolar depression (TRD). J Mental Health. 2010;19(2):168-75. 